\title{
STATUS OF THE LARGE HADRON COLLIDER (LHC)
}

\author{
F. Bordry, CERN, Geneva, Switzerland
}

\section{Abstract}

The installation of the LHC (Large Hadron Collider) at CERN is now completed. All magnets are interconnected. Five of the eight machine sectors are cold and filled with liquid helium. The remaining three sectors are in the cooldown phase. All the sectors will be at $1.9 \mathrm{~K}$ by mid of July 2008. The hardware commissioning has started in 2006 and three sectors are commissioned up to $5.5 \mathrm{TeV}$. The start of the beam commissioning is planned for this summer 2008. The paper presents the main milestones towards the beam operation and summarizes the major technical difficulties which were discovered and recovered during the past two years.

\section{INTRODUCTION}

The Large Hadron Collider (LHC) accelerator was originally conceived in the 1980s and approved for construction by the CERN Council in late 1994. The machine parameters, the $R \& D$ and technical validation together with progress of the production and installation were reported every year in EPAC and PAC status papers (e.g. [1] and [2]). Only a rapid summary of the LHC layout will be given in this introduction. More data can be found in the status papers and also in the numerous papers describing the different systems required for the LHC operation [3].

The LHC, installed in the $27 \mathrm{~km}$ long tunnel that had previously been used for LEP (Large Electron Positron accelerator), will provide collisions with a centre-of-mass energy of $14 \mathrm{TeV}$ and a luminosity of $10^{34} \mathrm{~cm}^{-1} \mathrm{~s}^{-2}$. The LHC has an 8-fold symmetry with eight arc sections, and eight straight sections (LSS) which contain experiments and systems for machine operation (Fig. 1). Two counterrotating proton beams will circulate in separate vacuum chambers installed in the twin-aperture magnets, with the beams crossing at four points. The beams will collide at a small angle in the centre of the main experimental detectors (ATLAS, ALICE, CMS and LHCb) installed in four of the straight sections. The injection elements are installed in the insertions where ALICE and LHCb are located. Of the remaining four insertions, two contain the beam collimation systems (LSS3 and LSS7), one contains the radio-frequency (RF) acceleration system (LSS4) and one contains the beam abort systems for the two beams (LSS6).

In the following, we will discuss the main milestones towards the beam operation (magnet interconnection, sector cool-down, collimators, beam dump, RF system, powering and transfer line test) and summarize the major technical difficulties which were discovered and recovered during the past two years: low- $\beta$ triplets and RF plug-in modules.

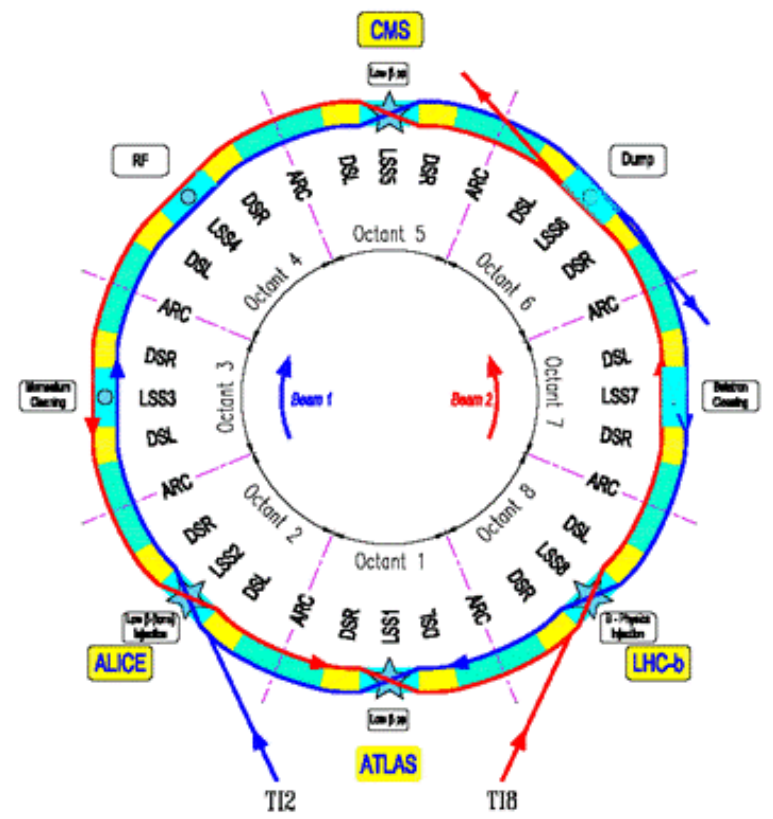

Figure 1: LHC machine general layout.

\section{STATUS OF THE INSTALLATION AND ASSEMBLY}

Three notable milestones were reached with the helium distribution line (QRL) finished in November 2006, the lowering of the last dipole magnet in April 2007 and the closure of the continuous cryostats in November 2007. The latter was the main assembly activity. It concerns the 1695 magnet-to-magnet and 224 magnet-to-cryogenic distribution line interconnects which require the interconnection of the beam vacuum, electrical, cryogenics and thermal insulation systems. Each magnet-to-magnet interconnection is a complex operation involving electrical assembly (ultrasonic welding, brazing) and mechanical assemblies, mainly welding but also soldering. Before passing to the mechanical assembly of the tubes containing the electrical connections a thorough test of the concerned electrical circuits was carried-out. The leak tightness of the assembly is verified on a subsector basis after the helium vessel is completely closed. Each magnet-to-magnet interconnect consists of 9 assembly actions, 5 leak tightness checks, 5 electrical tests and one $1 \mathrm{RF}$ test [4].

For each sector this totals to 1964 assembly interventions, 226 electrical tests, 70 vacuum tests and 14 $\mathrm{RF}$ test on sub-assemblies. The first interconnection started on May 2005 and the last one was completed at the end of 2007 (not taking the consolidation phases of sector 7-8 and sector 4-5 into account). 


\section{LHC SECTORS COOL-DOWN}

The LHC machine is composed of:

- main superconducting magnets in the arcs and the dispersion suppressors cooled in superfluid helium at $1.9 \mathrm{~K}$,

- stand alone superconducting magnets in the matching sections cooled in liquid helium at $4.5 \mathrm{~K}$,

- superconducting quadrupole strings in the inner triplets cooled at $1.9 \mathrm{~K}$.

Electrical feed boxes which contain the current leads interface the cold and warm electrical circuit distribution. The cooling is produced by eight large cryogenic plants (one per sector) installed in five cryogenic islands. Each plant is able to produce up to an equivalent capacity of $18 \mathrm{~kW}$ at $4.5 \mathrm{~K}$ as well as up to $2.4 \mathrm{~kW}$ at $1.8 \mathrm{~K}$. The helium is distributed in the tunnel via a compound cryogenic line (QRL), running in parallel to the magnets.

The installation of the cryogenic equipments was completed by the end of 2006 with the reception of the last QRL sector. In 2006, a QRL sector was cold commissioned in order to validate the thermo-mechanical performance of the line which was found to be according to the specification.

The complete commissioning of a sector includes:

- the cool-down from $300 \mathrm{~K}$ to $80 \mathrm{~K}$ using the $600 \mathrm{~kW}$ liquid nitrogen pre-cooler. This phase takes typically about 3 weeks and the main challenge is the logistics of the supply of liquid nitrogen as 1250 tons of nitrogen - i.e. up to 6 trailers per day - have to be managed during this period.

- the cool-down from 80 to $5 \mathrm{~K}$ using the turboexpanders of the $4.5 \mathrm{~K}$ helium refrigerator. This phase takes typically 2 weeks and the main challenge concerns the tuning of the refrigeration cycle in order to maximize the cooling capacity.

- the helium filling. This phase takes few days and during this period, up to 15 tons of helium has to be liquefied. During the first half of 2008, a delivery rate of up to 6 helium trailers per month has been required to fulfil the demand.

- the cool-down from 5 to $1.9 \mathrm{~K}$. This phase takes few days and the main challenge is the tuning of the 1.8 $\mathrm{K}$ refrigeration unit and its cold compressors which are for the first time coupled to the sector equipments.

- the tuning of the tunnel cooling loops. This phase takes typically one week and the main challenge is to get stable temperature, pressure and level conditions in the magnets and the current leads.

By the middle of, four sectors were in nominal operation; one sector completely filled was in final cooldown phase; two sectors were in the cool-down phase between 80 and $5 \mathrm{~K}$; and one sector was in the first phase of cool-down with an average temperature of $230 \mathrm{~K}$.

\section{CONSOLIDATION AND COMMISSIONING OF LOW- $\boldsymbol{\beta}$ TRIPLETS}

The LHC low- $\beta$ triplets were supplied by a collaboration between CERN, Fermilab, KEK and LBNL, that delivered eight sets of triplets and their feed-boxes, one for each side of the four LHC interaction regions. The installation of the first low- $\beta$ triplet in the LHC tunnel was completed in sector 7-8 in December 2005. In spite of extensive testing of individual magnets during production and after delivery, this was the first time that the triplet string could be tested as a single system. One of the important system tests is the pressure test, when the equipment experiences the same conditions that will occur during LHC operation.

During the pressure test of sector 7-8 in November 2006, the heat exchanger of the inner triplet collapsed. The failure was traced to a faulty design of the extremities of the heat exchanger tube. After examining the technical alternatives and their implications on the LHC planning, it was decided in January 2007 that new tubes should be manufactured and installed. The repair programme was launched and new tubes were available in March 2007. The tubes were installed without removing the magnets from the tunnel, and by the end of May 2007 all magnets were fitted with new heat exchangers.

The first triplet so repaired was pressure tested in March 2007. During this test, however, the internal support system of the cryo-magnets made of glass reinforced plastic (GRP) "spiders" failed resulting in damage to one of the magnets. This second failure of the triplets triggered wide public attention and concern for their mechanical integrity. A task force including experts from CERN and Fermilab was charged with proposing, validating and implementing consolidation actions for all the triplets. The experts concluded that the asymmetrical forces generated by the pressure thrust at the two extremities of the triplet were not properly compensated, so that an axial force of about 10 tons was transmitted to the internal GRP supports, which were designed mainly for transverse loads. Four metal cartridges, consisting of an Invar rod inserted in an Al tube, were installed in all the quadrupoles, which are located at the two extremities of the triplet (Q1 and Q3). The elasticity and thermal contraction of the cartridges limit the movement of the magnets inside their cryostats, while reducing the load on the GRP supports in the full temperature range from room temperature to $1.9 \mathrm{~K}$. The cartridges and other interventions were implemented and tested on magnets and feedboxes available on the surface, while all other triplets were repaired in the tunnel. The first triplet completed in the tunnel was successfully pressure tested in July 2007 in sector 8-1. The consolidation and testing of the remaining seven triplets continued till September 2007 , when all triplets were confirmed to be ready for cool-down as part of the standard LHC sector commissioning procedure. 
The powering of these mixed quadrupoles uses two nested high-current power converters: [8kA, 8V] and [6kA, 8V] (Fig. 2) [6].

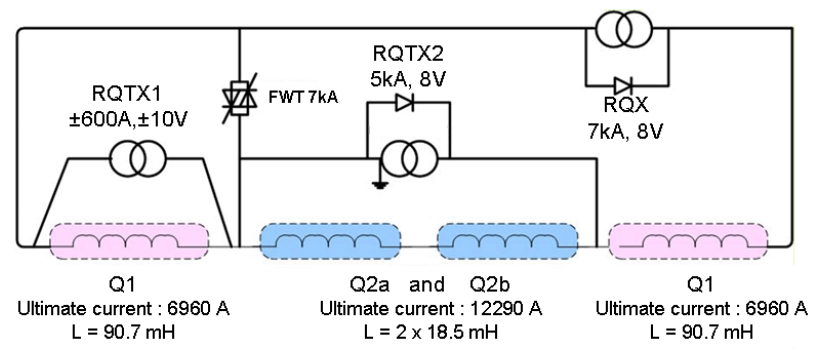

Figure 2: Powering principle of Inner Triplet Quadrupoles

The challenge of this circuit is to control the magnet currents with very high accuracy (1ppm, the same accuracy as for the main dipole and quadrupole circuits) in spite of the inductive coupling effect between the three power converters (variations of output voltage of one power converter have effects on all power converter currents). A special control strategy (decoupling control by state feedback) has been developed and implemented.

Successful powering of the low- $\beta$ triplet to nominal current was performed in sector 5-6 in April 2008 and in sector 7-8 in June 2008.

\section{COLLIMATOR SYSTEM STATUS}

The LHC is designed to store $360 \mathrm{MJ}$ in each of its two proton beams. This extends the state of the art in stored beam energy by 2-3 orders of magnitude. Small losses of stored beam cannot be avoided and a maximum loss rate of $0.1 \%$ per second has been specified. This corresponds to about $500 \mathrm{~kW}$ and must be safely absorbed. This must be compared with a quench threshold of $8.5 \mathrm{~W} / \mathrm{m}$ in the super-conducting LHC magnets. A multi-stage cleaning system has been designed [7] to intercept the beam losses in two dedicated warm cleaning insertions (LSS 3 and 7). The system relies initially on 88 two-sided, robust collimators, which are arranged for providing four stages of cleaning. The collimators have been installed and are being prepared for LHC operation. Synchronous and remotely controlled collimator movements have been demonstrated with the installed collimators, with independent position verification at the $30 \mu \mathrm{m}$ level. The system will be complemented with 28 collimators during the first shutdown (complete phase 1 system). It should then provide better than $99.99 \%$ of cleaning efficiency with collimation gaps down to $2-3 \mathrm{~mm}$. A further upgrade (phase 2) should extend the collimation performance by another factor 10, supporting a gradual increase in LHC intensity and luminosity. The production of the collimators, which was a major concern during 2006, is now well in hand and the deliveries were in line with the two-phase installation plan. The local hardware commissioning of the installed collimators is around $80 \%$ complete and the remote control has been successfully tested. Collimator jaw positioning and surveillance was demonstrated to within $30 \mu \mathrm{m}$ for the first 7 collimators (in LSS3) by executing a realistic 30 minute ramp function, fully meeting specification.

\section{RADIO FREQUENCY SYSTEM STATUS}

The RF accelerating system consists of sixteen 400 $\mathrm{MHz}$ SC cavities, four per cryomodule, and two modules per beam. Each cavity is powered by a $300 \mathrm{~kW}$ klystron. The klystrons are powered in groups of four by four 40A $60 \mathrm{kV}$ power converters installed on the surface. There are sophisticated feedback and beam control systems to minimize system noise and handle the high intensity LHC beams.

The transverse damping and feedback systems consist of electrostatic kickers, four per beam per plane. These are driven by tetrode amplifiers operating at up to $12 \mathrm{kV}$ at up to $20 \mathrm{MHz}$, again with power converters on the surface.

The damper and RF power systems were fully set up and tested at full power. The eight cavities connected to the sector 4-5 cryogenics systems, were conditioned to nominal voltage and provided each $2 \mathrm{MV}$ with a gradient of $5.5 \mathrm{MV} / \mathrm{m}$ at the beginning of 2008 . The remaining eight cavities (sector 3-4) are presently being filled with helium and will be powered in the coming weeks.

\section{BEAM DUMP SYSTEM STATUS}

The function of the beam dumping system is to fastextract the beam in a loss-free way from each ring of the collider and to transport it to an external absorber, positioned sufficiently far away to allow for appropriate beam dilution in order not to overheat the absorber material [8].

Installation of the beam dump system in LSS 6 is complete and the system commissioning tests almost finished. The full vacuum system has been baked where required and leak tested. All extraction and dilution kickers have been tested and calibrated at full strength and several months of data has been accumulated for the reliability run of the kicker switches. The fault-rate on the switches is in line with the expectations, confirming the high reliability of the generators. Integration into the overall controls environment for the dump system has been intensively driven by a series of dedicated dry runs.

\section{SUPERCONDUCTING CIRCUIT COMMISSIONING}

The sectorisation of the machine has been fully exploited for the hardware commissioning. Firstly the sector 7-8 and the sector 4-5 were cooled down to $1.9 \mathrm{~K}$ without the triplet magnets in order to gain experience of the cryogenic systems and to carry-out powering tests at cold. Sectors 7-8 (July 2007) and sector 4-5 (January 2008) were warmed up to connect the low- $\beta$ magnets to the QRL and for some consolidations. Then the cool down of the 8 sectors started according to the following sequence:

- 2007: sector 5-6 in November 
- 2008: sectors 7-8 in January 8, 8-1 in February, 6-7 in March, 2-3 and 3-4 in April, 1-2 and 4-5 in May

After the cool down to close to $1.9 \mathrm{~K}$ and before the powering tests can start, the electrical integrity of the superconducting circuits is verified as well as properties (resistance, inductance, transfer function,...) are measured. The powering tests which followed aimed at validating the interlock system which authorizes powering, the quench detection system as well as the energy extraction system for those circuits which have one. The aim of these tests is to validate the protection strategies under the different failure scenarios and to evaluate the behaviour of the magnet chain, the current leads and the power converters during a normal LHC ramp and steady state. These tests are repeated at different current levels. These validations are followed by the assessment of the performance of the power converters. The stability of the beam is dominated by the performance of the powering system. The segmentation of the LHC into eight sectors results in 24 main circuits instead of 3: eight main bending, focussing and defocusing circuits. A good tracking is vital for the LHC beam quality. The accuracy for these main circuits must be below 20ppm (part per million) and the reproducibility should be close to $5 \mathrm{ppm}$. The achievement of this high precision is certainly the most difficult challenge for the LHC power converters [9].

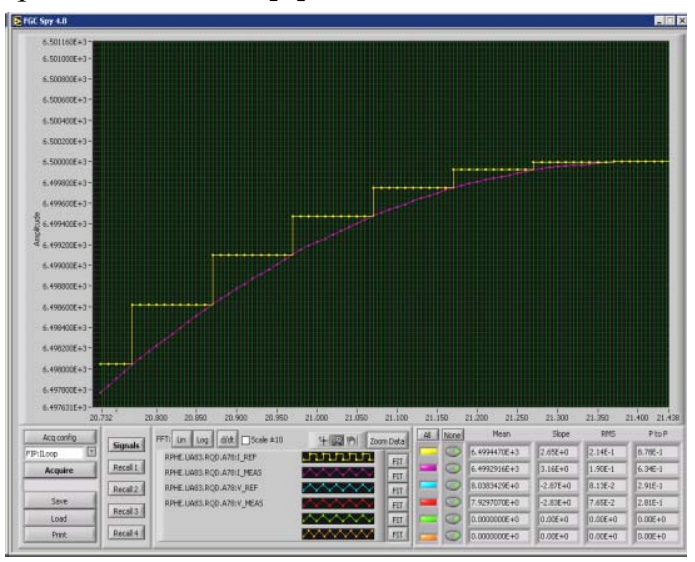

Figure 3: The end of the ramp at $6.5 \mathrm{kA}$ of the quadrupole converter with no lagging error and no overshoots

The first results of this campaign have validated the performance of the different types of power converters. The main achievements for the power converters were the optimization of the start-up algorithm to avoid triggering the quench protection system, the setting of the current loop parameters on large time constant loads, the validation of the current loop algorithm (no lagging error and no overshoot, Fig. 3) and the validation of the high precision performance.

The powering tests are completed with a long heat run which involve all the commissioned circuits. This aims at testing the stability of the converters but also of the associated infrastructure systems supplying electricity, cooling water and ventilation. For the three commissioned sectors, all the systems performed as expected. More detailed information on the hardware commissioning and its procedure are available in the dedicated paper presented at this conference [10].

Based on data from tests on individual magnets, the first natural quenches were expected to occur around $11 \mathrm{kA}(6.5 \mathrm{TeV})$; another 25 to 30 training quenches were anticipated for the 154 dipoles of each sector to reach nominal current $(11.85 \mathrm{kA}, 7 \mathrm{TeV})$. In order to meet the summer 2008 deadline for commissioning with beam a reduced beam energy $5 \mathrm{TeV}$ was proposed to the experiments and was accepted. Following this strategy, the hardware commissioning of the sectors 4-5 and 5-6 was completed up to $5.5 \mathrm{TeV}$. This strategy was confirmed to be adequate since the first quench on the sector 5-6 occurred at around 10kA and after almost 30 quenches the current reached is only $11.15 \mathrm{kA}(94 \%$ of nominal current). Further analyses are presently on going to understand the de-training effect observed on the dipoles of one of the manufacturers. Also strategies are studied to speed-up the training of all the sectors during the first beam shut-down.

\section{PLUG-IN MODULE PROBLEM}

After the warm-up of Sector 78, non-conformity was discovered: buckling of the RF fingers in the Plug-In Modules (PIMs). These PIMs ensure the electrical continuity of the beam screen between two magnets. Since the magnets contract during cool-down, the PIMs contain conducting "fingers" which must slide smoothly during cool-down and warm-up. For a small number of interconnects in Sector 7-8 (6 of the 366 modules), this was found not to be the case and a number of fingers buckled into the beam aperture. The problem is now understood: out of tolerances bending angles of the RF fingers and a slightly larger than nominal magnet gap between the main dipole and quadrupole transitions. A simple method has been developed to diagnose bad PIMs using a small ball with a $40 \mathrm{MHz}$ transmitter. The signals emitted by the transmitter are received by Beam Position Monitors (BPMs) located every 50 metres along the beam pipes, then recorded on a computer screen to allow the ball's progress through the pipe to be monitored. This method has been applied also to the sector 4-5 after its warming and to all sectors before their cool-down (except for the sector 5-6 which was already in the cool-down phase). In the future, this method will be applied systematically to each sector warmed up to room temperature and the faulty modules will be replaced.

\section{TRANSFER LINES TESTS}

The two SPS-to-LHC beam transfer lines TI2 and TI8 have a combined length of $5.6 \mathrm{~km}$ and comprise some seven hundred warm magnets. TI 2 will transport beam from SPS to the injection into LHC Ring 1 (LSS 2) and has first been tested with beam in October 2007. As for the other transfer line TI 8 (which supplies beam to LHC Ring 2) three years before [11], already the first shot 
passed down the entire line without any threading (Fig. 4). After initial tuning, a range of measurements was carried out with a low intensity proton beam, confirming the basic design and operability of this new long transfer line, and the expectations in terms of physical aperture, energy acceptance, and optics parameters. In view of the first injections into $\mathrm{LHC}$, the beam commissioning of the TI 2 and TI 8 lines, was resumed with new tests in May and June 2008.

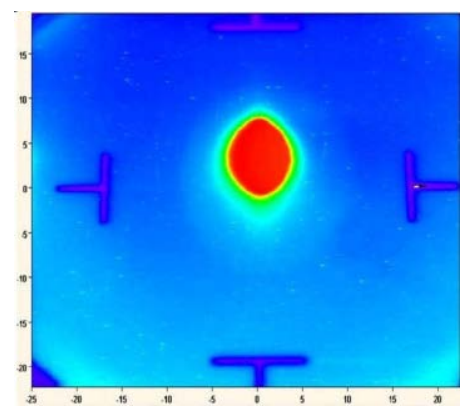

Figure 4: Image of the first beam spot on the last screen traversed by the beam during the TI 2 test in 2007 .

\section{BEAM COMMISSIONING PHASES}

The various challenges for the LHC beam operation are tackled by deploying a staged commissioning approach [12]. Following the overall strategy defined and confirmed after the discovery of the magnet de-training, the machine will be commissioned for operation at $5 \mathrm{TeV}$ per beam. Given reasonable efficiency for operations, it is estimated to take around 2 months elapsed time from first beam to first collisions. It is then envisaged to interleave physics runs with pushing machine performance. Table 1 summarises a possible evolution of key parameters for the first few weeks of physics running in late 2008. For 2009, it is hoped that the dipole magnets will have all been retrained for operation at or close to $7 \mathrm{TeV}$ per beam. Commissioning and operation would then follow the stages described in [12].

Table 1: Parameter evolution for early physics

\begin{tabular}{|c|c|c|c|c|}
\hline \multicolumn{3}{|c|}{ Parameters } & \multicolumn{2}{c|}{ Rates in 1 and 5 } \\
\hline $\begin{array}{c}\text { Bunches } \\
\text { per } \\
\text { beam }\end{array}$ & $\begin{array}{c}\text { Bunch } \\
\text { intensity }\end{array}$ & $\begin{array}{c}\beta^{*} \\
(\mathrm{~m})\end{array}$ & $\begin{array}{c}\text { Luminosity } \\
\left(\mathrm{cm}^{-2} \mathrm{~s}^{-1}\right)\end{array}$ & $\begin{array}{c}\text { Events/ } \\
\text { crossing }\end{array}$ \\
\hline $1(2)$ & $10^{10}$ & 11 & $1.110^{27}$ & $<<1$ \\
\hline 43 & $10^{10}$ & 11 & $5.010^{28}$ & $<<1$ \\
\hline 43 & $410^{10}$ & 11 & $8.010^{29}$ & $<<1$ \\
\hline 43 & $410^{10}$ & 3 & $2.910^{30}$ & 0.36 \\
\hline 156 & $410^{10}$ & 3 & $1.010^{31}$ & 0.36 \\
\hline 156 & $910^{10}$ & 3 & $5.410^{31}$ & 1.8 \\
\hline
\end{tabular}

\section{CONCLUSION}

Five of the eight sectors are cold and filled with liquid helium. The remaining three sectors are in the cool-down phase. All the sectors will be at $1.9 \mathrm{~K}$ by mid July 2008 . The hardware commissioning has started in 2006 and three sectors are commissioned up to $5.5 \mathrm{TeV}$ following the strategy which was adopted to gain time with the training of magnets.

As large and complex project, LHC is not all plain sailing but CERN has shown an impressive reactive force to overcome the obstacles and continued progressing towards its target of completing the LHC for physics. All the commissioning campaigns, which took place on many systems, have demonstrated that the systems perform as expected. If no further problems are encountered during the powering of the sectors, the whole ring will be ready to allow injecting beam by early August 2008. If, however, problems are encountered which require a full or partial warm-up of a sector to fix them (the most likely being an electrical fault or vacuum leak), the schedule can slip by two to three months.

The commissioning and the operation of the LHC machine are and will be an absorbing and captivating unique period.

\section{ACKNOWLEDGMENTS}

The paper has been written on behalf of all CERN staff participating to the LHC project, as well as all those involved from external collaborations and industries. Special thanks are due to J.P.Tock, L.Tavian, R.Ostovic, R.Assmann, R.Saban, E.Ciapala, B.Goddard, V.Mertens, and RBailey for all the documentation and information they provided.

\section{REFERENCES}

[1] O. Brüning, "LHC Progress and commissioning plans", EPAC 2006, Edinburgh, Scotland

[2] L. Evans, "LHC: construction and commissioning status", Pac07, Albuquerque, New Mexico, USA

[3] http://www.jacow.org/

[4] J.P. Tock et al., "The Interconnections of the LHC Cryomagnets at CERN", Magnet Technology 20, Philadelphia, 2007

[5] V. Benda et al., "Conceptual design of the cryogenic system for the LHC", EPAC'96, Sitges, Spain

[6] F. Bordry, H. Thiesen, "LHC Inner Triplet powering strategy", PAC 2001, Chicago, Illinois, U.S.A.

[7] R. Assmann et al. "The Final Collimation System for the LHC", EPAC 2006, Edinburgh, Scotland

[8] R. Schmidt et al., "Protection of the CERN Large Hadron Collider", New Journal of Physics 8, 2006

[9] F. Bordry, "Development and production of the Large Hadron Collider powering system", PCIM Conference, Nurnberg - Germany, June 2005

[10]R. Saban, "LHC Hardware Commissioning Summary", EPAC 2008, Genoa, Italy

[11] J.Uythoven et al., "Commissioning of the LHC Beam Transfer Line TI 8", PAC2005, Knoxville, USA

[12]R. Bailey et al., "A Staged Approach to LHC Commissioning”, EPAC 2006, Edinburgh, Scotland 